\title{
ESTUDIO EXPLORATORIO DE LOS LAMINADOS DE BAMBÚ GUADUA ANGUSTIFOLIA COMO MATERIAL ESTRUCTURAL
}

\author{
EXPLORATORY STUDY OF THE GLUED LAMINATED BAMBOO \\ GUADUA ANGUSTIFOLIA AS A STRUCTURAL MATERIAL
}

Luis Felipe López', Juan Francisco Correal ${ }^{2}$

\begin{abstract}
RESUMEN
Este artículo presenta los resultados de un estudio experimental, desarrollado en la Universidad de los Andes en Bogotá D.C., Colombia, acerca del potencial de los laminados de guadua (Guadua angustifolia Kunt) como material estructural. La influencia de la dirección de las fibras y de la ubicación de las láminas de guadua en las propiedades mecánicas es discutida en detalle. Los esfuerzos de diseño a compresión, tracción, corte y flexión de los laminados de guadua son comparados con los respectivos esfuerzos de las maderas estructurales Andinas. Además, el efecto en la resistencia al corte del adhesivo cuando el laminado de guadua es sometido a diferentes temperaturas de ensayo es estudiado. Basado en los resultados obtenidos del programa experimental desarrollado en este estudio y desde el punto de vista de sus propiedades mecánicas, se puede concluir que los laminados de guadua se proyectan como un material alternativo para la construcción de estructuras.
\end{abstract}

Palabras Claves: Guadua, bambú, laminados, propiedades mecánicas, materiales sostenibles.

\begin{abstract}
An exploratory study, conducted at Universidad de los Andes in Bogotá, Colombia, of the potential use of glued laminated bamboo guadua (Guadua angustifolia Kunt) as a structural material is presented in this paper. The influence of the direction of the fiber and the location of the laminae in the mechanical properties is discussed. The compression, tension, shear and flexural design stresses of glued laminated guadua are compared to those of Andean structural woods (lumber). Additionally, the effect on the mechanical properties of the bond strength of the adhesive at different test temperatures is investigated. Based on the mechanical properties obtained in this research, glued laminated guadua bamboo can be a suitable construction material for structures.
\end{abstract}

Keywords: Guadua, bamboo, laminated, mechanical properties, sustainable materials

\footnotetext{
${ }^{1}$ Ingeniero Investigador, Departamento de Ingeniería Civil y Ambiental,

Facultad de Ingeniería Universidad de los Andes, Bogotá D.C. Colombia. luisfl@yahoo.com

${ }^{2}$ Director del Laboratorio Integrado y Profesor Asistente del Departamento de Ingeniería Civil y Ambiental,

Universidad de los Andes, Bogotá, Colombia

Autor para correspondencia: jcorreal@uniandes.edu.co

Recibido: 16.02.2009. Aceptado: 31.07.2009
} 


\section{INTRODUCCIÓN}

Los bambúes han sido utilizados desde hace siglos en construcciones tradicionales especialmente en países como China y Japón. Esto se debe principalmente a su excelente relación resistencia-peso, alta sostenibilidad debido a su velocidad de crecimiento (3 a 6 años) y flexibilidad arquitectónica para la creación de diferentes formas geométricas. Además los bambúes tienen una ventaja adicional, que en la actualidad es cada vez más relevante para el medio ambiente, como es la capacidad para la captación de $\mathrm{CO}_{2}$. De acuerdo con INBAR (International network for bamboo and rattan 2007), en el mundo existen alrededor de 1250 especies de bambú, localizadas principalmente en América, África y Asia. En América hay cerca de 600 especies diseminadas desde el sur de los Estados Unidos hasta el sur de Chile y Argentina, desde los $0 \mathrm{msnm}$ hasta los $3000 \mathrm{msnm}$. No obstante, la gran diversidad de especies de bambú, solo 20 de estas han sido estudiadas y se consideran aptas para aplicaciones estructurales.

En América una de las especies más usada en la construcción de estructuras vernáculas es la Guadua angustifolia Kunt. Esta especie pertenece a la familia de las Poaceae y sobresale por el gran tamaño de sus culmos, los cuales alcanzan hasta 25 metros de altura con diámetros que oscilan entre 10 y $18 \mathrm{~cm}$. En Colombia existen cerca de 35.000 hectáreas de Guadua angustifolia kunt (Ministerio de Agricultura y Desarrollo Rural de Colombia 2006), ubicadas en los departamentos de Caldas, Risaralda, Quindío y Valle del Cauca principalmente. Investigaciones previas en guadua, Martin y Mateus (1981), Gómez y Rubio (1990), Prieto y Sanchez (2002), han reportado que existe una variación importante de las propiedades mecánicas en su estado rollizo, debido principalmente a la irregularidad dimensional de la sección transversal, lo cual restringe su uso en estructuras de grandes luces y cargas. Por lo anterior y teniendo en cuenta las ventajas que presentan los bambúes, los laminados de guadua se presentan como una alternativa para el uso de este material en la construcción de estructuras para las cuales la guadua es su estado rollizo presenta limitaciones.

En el mundo se han realizado muy pocas investigaciones sobre laminados de bambú. La primera investigación Lee et al. 1998, consistió en determinar experimentalmente la resistencia de vigas elaboradas con láminas de moso bambú, proveniente de una plantación del estado de Carolina del Sur en los Estados Unidos. En este estudio, se determinó la variación de la resistencia mecánica a flexión de laminados fabricados con diferentes dosificaciones de adhesivo y para diferentes contenidos de humedad. Adicionalmente, se estableció la resistencia al corte del adhesivo ureaformaldehido y resorcinol ureaformaldehido usado en los laminados de moso bambú. Además del estudio anterior, se han desarrollado investigaciones Nugroho y Ando (2000) y Nugroho y Ando (2001) de vigas compuestas por capas de esterillas de moso bambú con diferentes dosificaciones de adhesivo, humedades y densidades. En Colombia se han efectuado solo dos investigaciones Barreto (2003) y Duran (2003) en laminados de guadua, en las cuales se estudio la resistencia al corte en la línea de adhesivo y la resistencia a la compresión paralela a la fibra para diferentes tipos de adhesivo.

Basado en los resultados positivos de estas investigaciones y con el propósito de estimar cual es el potencial de los laminados de guadua para aplicaciones estructurales, se desarrollo en el presente estudio, una investigación de tipo experimental con el fin de determinar sus propiedades mecánicas. Las propiedades evaluadas en este estudio son: la resistencia a compresión y tensión tanto paralela como perpendicular a la fibra, la resistencia al corte paralelo a la fibra y la resistencia a flexión. Adicionalmente, se exploró la variabilidad de la resistencia al corte en la línea de adhesivo a diferentes temperaturas de ensayo para determinar su influencia en la resistencia del adhesivo tipo polivinilo de acetato (PVA). 


\section{MATERIALES Y MÉTODOS}

\section{Construcción de probetas}

Los laminados de guadua fueron producidos por la empresa Colguadua Ltda., la cual se encuentra ubicada en el municipio de Caicedonia Valle del Cauca, Colombia. Para la fabricación de los laminados de guadua se utilizó adhesivo de tipo polivinilo de acetato (PVA). Este tipo de adhesivo no es recomendado para ser usado en laminados estructurales, debido principalmente a su inestabilidad ante cambios importantes de temperatura. Teniendo en cuenta que este estudio se enfoca en explorar el potencial del material desde el punto de vista de su resistencia y no de su durabilidad, y sabiendo que la caracterización mecánica no se va a desarrollar bajo condiciones que afecten el comportamiento del adhesivo, se decidió adelantar la fase experimental con el adhesivo PVA.
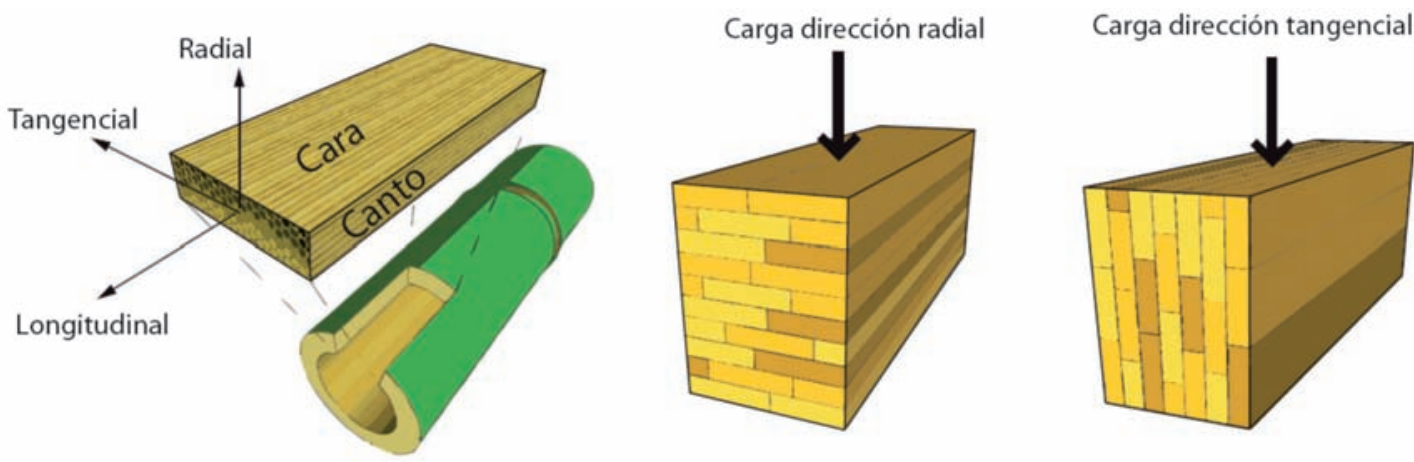

Figura 1. Distribución de láminas de guadua

Todos los culmos de la especie Guadua angustifolia Kunt que se utilizaron en la producción de las probetas tenían más de 4 años de edad y fueron cortados de plantaciones ubicadas en el municipio de Caicedonia en el departamento del Valle del Cauca, Colombia a $1300 \mathrm{msnm}$. Una vez que las guaduas son cortadas en tramos de $1 \mathrm{~m}$, pasan a la planta de producción de laminados en donde se elaboran las probetas a partir de láminas de $6 \mathrm{~mm}$ x $20 \mathrm{~mm}$ x $1000 \mathrm{~mm}$ (Figura 1). Una vez retirada la corteza exterior de la guadua y los tabiques interiores, las láminas son preservadas con una solución hidrosoluble con fungicida para el control del hongo cromógeno durante el secado en horno, el cual se realiza a una temperatura de $70{ }^{\circ} \mathrm{C}$ hasta alcanzar un contenido de humedad del $5 \%$. Las láminas secas son cepilladas por sus cuatro lados y luego pasan a la maquina encoladora de rodillos que dosifica la cantidad de adhesivo aplicado, $200 \mathrm{~g} / \mathrm{m}^{2}$ en las caras de las laminas y $150 \mathrm{~g} / \mathrm{m}^{2}$ en los cantos. Éstas son usadas para conformar tableros de láminas adheridas por sus cantos con una presión de $0.8 \mathrm{MPa}$. Finalmente, estos tableros son usados para construir la pieza de laminado aplicando una presión de 1.2 $\mathrm{MPa}$. El proceso de prensado se realiza a una temperatura ambiente promedio de $22^{\circ} \mathrm{C}$. Para estas condiciones ambientales, el tiempo de curado abierto es de 10 minutos y el tiempo de curado cerrado o tiempo de prensado es de 5 minutos. Finalmente, el producto terminado es sometido a una inmersión en una solución oleosoluble, con base en insecticidas piretroides, con concentraciones que oscilan entre el $1 \%$ y $3 \%$, para completar el proceso de preservación. 


\section{Procedimiento de Ensayo}

Los ensayos se realizaron en el Laboratorio de Materiales y Modelos Estructurales de la Universidad de los Andes, en Bogotá D.C. Colombia. El programa experimental se desarrolló con una máquina universal de pruebas tipo MTS Digital Control System (Test Start II). Debido a la falta de normas técnicas para realizar ensayos de caracterización en laminados de bambú, se adoptaron los procedimientos de ensayos de las normas técnicas para madera de la ASTM y del ICONTEC, que editan la Norma Técnica Colombiana (NTC). Los ensayos de caracterización física y mecánica realizados y las normas asociadas se listan en la Tabla 1.

Tabla 1. Ensayos de caracterización física y mecánica

\begin{tabular}{|c|c|c|c|c|}
\hline Nombre del Ensayo & $\begin{array}{c}\text { Numero de } \\
\text { Probetas }\end{array}$ & \multicolumn{2}{|c|}{ Norma Técnica del Ensayo } & Resultado Reportado \\
\hline Densidad & 131 & ASTM D2395 - 93 & NTC 290 & Masa por volumen \\
\hline Contenido de humedad & 213 & ASTM D 143-94 & NTC 206-1 & Contenido de humedad \\
\hline Corte en línea de adhesivo & 47 & $\begin{array}{c}\text { ASTM D 905 } \\
\text { ASTM D 1037 }\end{array}$ & NTC 2279 & Esfuerzo cortante máximo \\
\hline Tracción paralela a las fibras & 30 & ASTM D 3500-90 & $\begin{array}{c}\text { Esfuerzo máximo y } \\
\text { modulo de elasticidad }\end{array}$ \\
\hline Tracción perpendicular a las fibras & 17 & ASTM D 143-94 & $\begin{array}{c}\text { NTC 961 } \\
\text { NTC 3377 }\end{array}$ & $\begin{array}{c}\text { Esfuerzo máximo } \\
\text { (radial y tangencial) }\end{array}$ \\
\hline Compresión paralela a las fibras & 33 & ASTM D 143-94, & $\begin{array}{c}\text { NTC 784 } 3377 \\
\text { NTC límite proporcional y modulo } \\
\text { de elasticidad }\end{array}$ \\
\hline Compresión perpendicular a las fibras & 33 & ASTM D 143-94 & $\begin{array}{c}\text { NTC 785 } \\
\text { NTC 3377 }\end{array}$ & $\begin{array}{c}\text { Esfuerzo en el límite propor- } \\
\text { cional (radial y tangencial) }\end{array}$ \\
\hline Corte paralelo a las fibras & 33 & ASTM D 143-94 & $\begin{array}{c}\text { NTC 775 } \\
\text { NTC 3377 }\end{array}$ & $\begin{array}{c}\text { Esfuerzo máximo, esfuerzo en } \\
\text { Esfúximo }\end{array}$ \\
\hline Flexión & 33 & ASTM D 198 & ASTM D 143-94 & NT63 \\
\hline
\end{tabular}

\section{RESULTADOS Y DISCUSION}

\section{Densidad y contenido de humedad}

De acuerdo a los resultados de las probetas ensayadas, se determinó que la densidad de la guadua laminada es en promedio de $715 \mathrm{~kg} / \mathrm{m}^{3}$, con una desviación estándar de $31 \mathrm{~kg} / \mathrm{m}^{3}$ y un coeficiente de variación de $4.7 \%$. Esta densidad corresponde a un contenido de humedad promedio de $12 \%$, consistente con las condiciones medioambientales del laboratorio. 
Tabla 2. Comparación de la densidad de la guadua laminada con otras especies

\begin{tabular}{|l|l|c|}
\hline Nombre común & Nombre científico & $\begin{array}{c}\text { Densidad } \\
{\left[\mathbf{k g} / \mathbf{m}^{3}\right]}\end{array}$ \\
\hline Bambú gigante & Phyllostachys bambusoides & 480 \\
\hline Madera pino radiata & Pinus radiata & 500 \\
\hline Madera laminada, Southern Pine & Pinus radiata & 505 \\
\hline Madera laminada, Douglas Fir & Pseudotsuga menzresii & 530 \\
\hline Tulda & Bambusa tulda & 640 \\
\hline Cacutta bambú & Cacutta bambú & 661 \\
\hline Guadua laminada & Guadua angustifolia Kunt & $\mathbf{7 1 5}$ \\
\hline Moso bambú laminado & Phyllostachys pubencens & 720 \\
\hline Bambusa & Bambusa vulgaris & 790 \\
\hline Madera sapan & Clathrotropis brachypetala & 820 \\
\hline
\end{tabular}

Al comparar la densidad de los laminados de guadua con otras especies de bambú laminado y no laminado (Tabla 2), se puede observar que estos presentan densidades relativamente altas con excepción del moso bambú laminado y la bambusa, los cuales tienen densidades superiores en $1 \% \mathrm{y}$ $10 \%$, respectivamente. Para el caso particular de la bambusa, su alto contenido de almidón hace que este bambú necesite consideraciones especiales de inmunización reduciendo su potencial como material estructural debido a los costos adicionales que este proceso implica. La comparación de los laminados de guadua con algunas especies de madera como el pino radiata, sapan y maderas laminadas, indican que los laminados de guadua pueden considerarse dentro del rango de maderas densas. La densidad de los laminados de guadua es un buen indicador de su rigidez, parámetro de mayor importancia en el dimensionamiento y diseño de elementos estructurales.

\section{Propiedades Mecánicas}

Debido a la distribución particular de las láminas de guadua o bambú en los laminados, es posible obtener para una sección transversal sometida a un esfuerzo específico dos diferentes direcciones (radial y tangencial) de las láminas, las cuales se muestran en la figura 1. En la tabla 3 se muestran los resultados de las propiedades mecánicas de los laminados de guadua obtenidas de los ensayos de laboratorio. Es esta tabla se muestra el promedio, desviación estándar (D.E) y el coeficiente de variación (C.V) para cada una de las propiedades evaluadas. En promedio se obtuvo un coeficiente de variación del $15 \%$, lo cual es característico de materiales naturales. 
Tabla 3. Resumen de resultados de propiedades mecánicas de laminados de guadua

\begin{tabular}{|c|c|c|c|}
\hline Propiedades Mecánicas & Promedio (MPa) & $\begin{array}{l}\text { D.E } \\
\text { (MPa) }\end{array}$ & $\underset{\%}{\text { C.V }}$ \\
\hline \multicolumn{4}{|l|}{ Compresión paralela a la fibra. } \\
\hline Esfuerzo máximo & 48.0 & 3.0 & $5 \%$ \\
\hline Esfuerzo en el límite proporcional & 36.0 & 2.0 & $6 \%$ \\
\hline Modulo de elasticidad & 19137 & 1625 & $9 \%$ \\
\hline \multicolumn{4}{|l|}{ Compresión perpendicular a la fibra } \\
\hline Esfuerzo máximo radial & 5.0 & 0.6 & $12 \%$ \\
\hline Esfuerzo máximo tangencial & 6.8 & 0.9 & $13 \%$ \\
\hline \multicolumn{4}{|l|}{ Tracción paralela a la fibra. } \\
\hline Esfuerzo máximo & 132.0 & 24.1 & $18 \%$ \\
\hline Módulo de elasticidad & 17468 & 3655 & $21 \%$ \\
\hline \multicolumn{4}{|l|}{ Tracción perpendicular a la fibra } \\
\hline Esfuerzo máximo radial & 1.1 & 0.3 & $22 \%$ \\
\hline Esfuerzo máximo tangencial & 1.8 & 0.4 & $21 \%$ \\
\hline \multicolumn{4}{|l|}{ Corte paralela a la fibra } \\
\hline Esfuerzo máximo & 9.4 & 1.2 & $13 \%$ \\
\hline \multicolumn{4}{|l|}{ Flexión } \\
\hline Esfuerzo máximo radial & 74.0 & 10.6 & $14 \%$ \\
\hline Módulo de elasticidad radial & 9523 & 1100 & $12 \%$ \\
\hline Esfuerzo máximo tangencial & 87.0 & 12.8 & $15 \%$ \\
\hline Módulo de elasticidad tangencial & 11456 & 1450 & $13 \%$ \\
\hline
\end{tabular}

D.E = Desviación Estándar y C.V = Coeficiente de Variación

El valor del esfuerzo máximo a compresión en laminados de guadua es dependiente de la dirección de las fibras. El esfuerzo máximo a la compresión paralela a la fibra es 7 y 10 veces mayor que el esfuerzo máximo a la compresión perpendicular a la fibra en las direcciones radial y tangencial respectivamente. Esta diferencia se presenta principalmente por la contribución de las fibras, las cuales en el ensayo de compresión paralela se encuentran en la misma dirección de la carga y confinadas por la matriz (parénquima). En el caso de la compresión perpendicular a las fibras existe una diferencia en el esfuerzo máximo, dependiendo si la carga se aplica en dirección radial o tangencial. De acuerdo a lo anterior, el esfuerzo máximo en la dirección tangencial es mayor en un 36\% con respecto al esfuerzo en la dirección radial. La distribución de las fibras puede producir este tipo de diferencia en los esfuerzos máximos de compresión perpendicular, ya que la concentración de estas es mayor en la periferia de la guadua (Figura 2) y el arreglo de las láminas en el sentido radial define capas de diferente densidad que inducen fallas por aplastamiento a esfuerzos menores.

El esfuerzo a la tracción es influenciado de una manera más clara por la dirección de las fibras que el esfuerzo a compresión. El esfuerzo a tracción máximo en dirección paralela a la fibra es entre 73 y 120 veces mayor que en la dirección perpendicular. Esta diferencia es debido a la capacidad de 
las fibras de guadua para resistir esfuerzos de tracción y a su continuidad a todo lo largo del culmo. El esfuerzo máximo a tracción perpendicular fue determinado tanto para la dirección radial como tangencial. En la Tabla 3, se observa que el esfuerzo máximo de tracción perpendicular a la fibra en la dirección tangencial es 64\% mayor que el radial. Durante los ensayos, se notó que la falla del ensayo de tracción perpendicular a la fibra radial era controlada por la adherencia del adhesivo, mientras que la falla del ensayo de tracción tangencial era debida a una combinación de falla del adhesivo y parénquima lo cual explica el incremento del esfuerzo último.

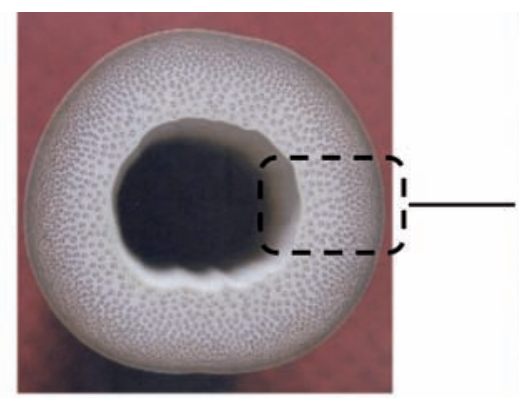

(a)Sección transversal

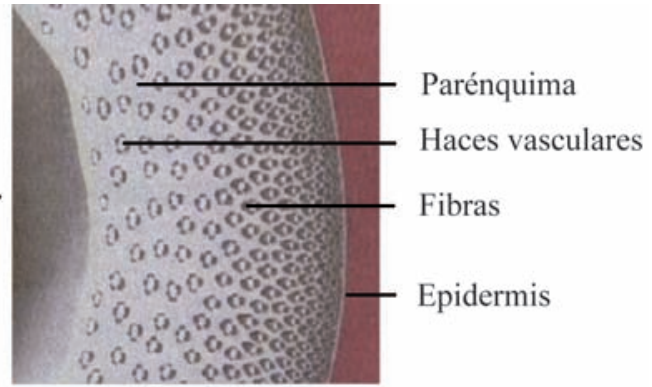

(b)Pared del culmo de bambú

Figura 2. Distribución de las fibras de guadua en el espesor de la pared

Con el propósito de determinar la influencia de la dirección de las láminas en el esfuerzo de flexión, se ensayaron probetas con orientación de láminas radial y tangencial. El esfuerzo máximo a flexión es mayor en $18 \%$ con las láminas en dirección tangencial a la carga con respecto al esfuerzo de flexión máximo con láminas radiales a la carga. Esta diferencia fue del mismo orden en los módulos de elasticidad a flexión para cada una de las direcciones tangencial y radial. Este aumento del esfuerzo máximo de flexión, se produce debido la mejor disposición de las láminas tangenciales para resistir los esfuerzos que se producen en la sección debido a la flexión. En las láminas radiales se tiene una línea de adhesivo a todo lo ancho de sección (Figura 1), lo cual puede inducir fallas por delaminación debido a los esfuerzos cortantes horizontales. Este tipo de falla se pudo apreciar en los ensayos a flexión con láminas cargadas de forma radial.

El percentil 5, (P5) de las propiedades mecánicas de los laminados de guadua, fue calculado con el fin de establecer una comparación con los valores de diseño para los diferentes grupos de maderas estructural clasificadas en el Manual de diseño para maderas del grupo Andino (Junta del Acuerdo de Cartagena 1984) y que sirvió como base para la elaboración del Titulo G (estructuras de madera) de la Norma Colombiana de Diseño y Construcción Sismo-Resistente (NSR-98) (Asociación Colombiana de Ingeniería Sísmica 1997). La Figura 3, muestra la densidad y los valores del P5 de las propiedades mecánicas fundamentales para la guadua rolliza (Lopez y Silva 2000) y (Lopez y Trujillo 2002), los laminados de guadua y los grupos de madera estructural según la NSR-98 (Asociación Colombiana de Ingeniería Sísmica 1997). El eje vertical de la Figura 3 fue normalizado con respecto al menor valor entre la guadua y las maderas estructurales. Para la densidad y cada una de las propiedades mecánicas, se puede observar, que la guadua laminada presenta una resistencia de diseño (percentil 5) más alta que las maderas estructurales Andinas más resistentes (Grupo A) y la guadua rolliza. En particular, los laminados son un $27 \%$ más resistentes en corte paralelo y flexión y un $47 \%$ más resistentes en compresión paralela que el Grupo A de maderas estructurales. En cuanto a la resistencia a la tensión de diseño, los laminados de guadua son 180 \% más resistentes que el Grupo A de maderas. Es importante resaltar que los laminados tienen una densidad comparable con las maderas del Grupo A, pero su resistencia es considerablemente mayor. 


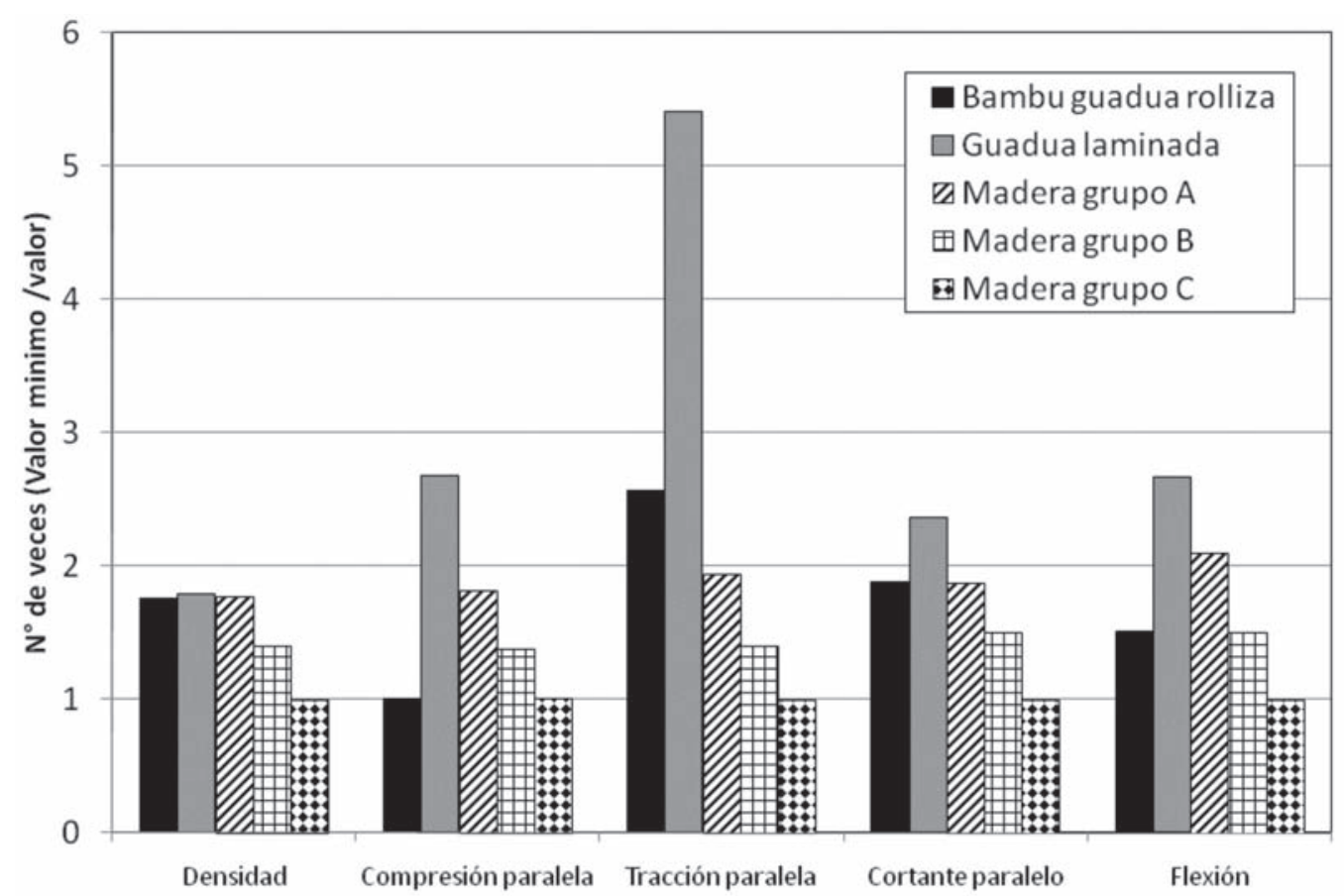

Figura 3. Comparación entre guadua rolliza, laminados de guadua y maderas estructurales Colombianas

\section{Resistencia del adhesivo en laminados de guadua}

La resistencia del adhesivo usado en los laminados, se evaluó mediante el ensayo del corte en la línea de adhesivo. En la Tabla 4 se lista el esfuerzo al corte del adhesivo obtenido en esta y otras investigaciones (Barreto 2003) y (Durán 2003) de laminados de guadua. Como se puede ver, el esfuerzo al corte obtenido en esta investigación con PVA es de 2 y 1.4 veces mayor que con el adhesivo tipo urea formaldehído y polycloropreno, respectivamente. Adicionalmente, se aprecia una diferencia de $46 \%$ entre las resistencias al corte con adhesivos PVA. Estas diferencias pueden estar asociadas al proceso de fabricación, ya que los tiempos de curado, la cantidad de adhesivo y el prensado aplicado son fundamentales para lograr una óptima adhesión entre las superficies.

Tabla 4. Esfuerzo de corte en línea de adhesivo

\begin{tabular}{|l|c|c|}
\hline \multicolumn{1}{|c|}{ Autor } & Tipo de Adhesivo & Esfuerzo de Corte (MPa) \\
\hline Barreto (2003) & Urea formaldehído & 3.91 \\
\hline \multirow{3}{*}{ Durán (2003) } & Urea formaldehído & 3.01 \\
\cline { 2 - 3 } & Polycloropreno & 5.67 \\
\cline { 2 - 3 } & PVA & 4.22 \\
\hline López y Correal (en el presente estudio) & PVA & 7.92 \\
\hline
\end{tabular}


Con el fin de conocer el comportamiento del adhesivo tipo PVA ante diferentes temperaturas, el ensayo de corte en línea de adhesivo además de ser realizado en la temperatura promedio del laboratorio $\left(20^{\circ} \mathrm{C}\right)$ fue efectuado también a $30^{\circ} \mathrm{C}, 50^{\circ} \mathrm{C}$ y $70^{\circ} \mathrm{C}$. En la Figura 4 se muestran los valores promedio del esfuerzo máximo al corte en la línea de adhesivo para las diferentes temperaturas consideradas. La mayoría de los ensayos presentaron falla de cohesión, ya que la resistencia del adhesivo nunca fue superior a la resistencia al corte paralelo a la fibra de los laminados. Existe una tendencia de la resistencia del adhesivo a disminuir cuando la temperatura aumenta. Esta disminución es del orden de $35 \%$ con respecto a la resistencia a temperaturas de $20^{\circ} \mathrm{C}$ y $30^{\circ} \mathrm{C}$. Una vez realizados los ensayos de corte a $50^{\circ} \mathrm{C}$ y $70^{\circ} \mathrm{C}$ se observó que el adhesivo presentaba fluencia, es decir un ablandamiento progresivo con el aumento de temperatura lo cual es típico en este tipo de adhesivos denominados termoplásticos. Este comportamiento lo hace poco recomendable para uso como adhesivo estructural.

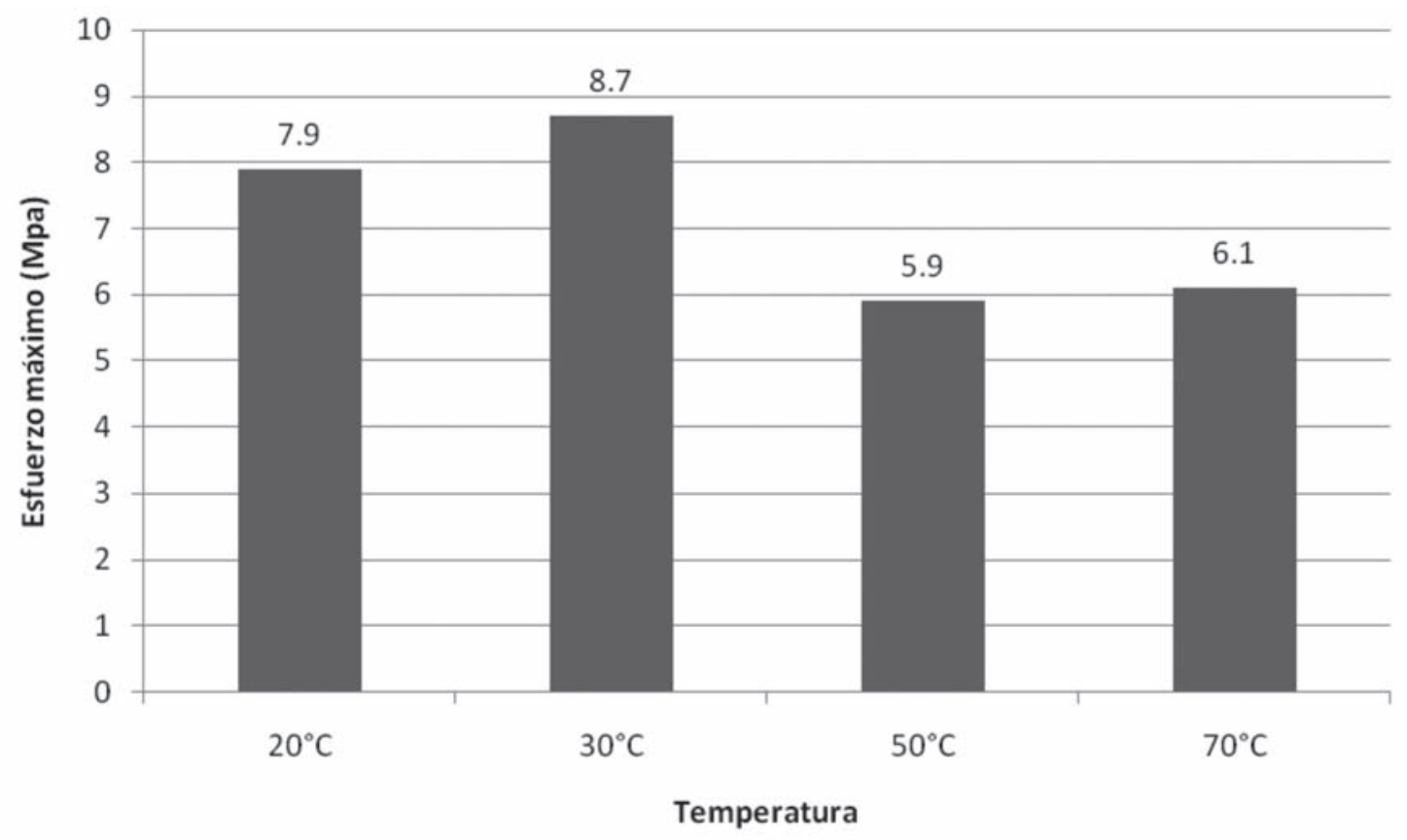

Figura 4. Resultado esfuerzo de corte en línea de adhesivo a diferente temperatura.

\section{CONCLUSIONES Y RECOMENDACIONES}

Basado en los resultados experimentales de esta investigación y teniendo en cuenta el análisis de estos se puede concluir:

La dirección de las fibras en los esfuerzos de compresión y de tracción afectan su capacidad de resistencia. Por lo tanto, es recomendable en lo posible, orientar los elementos de tal manera que la carga sea aplicada paralela a la fibra.

La sección transversal de los laminados de guadua usados como elementos sometidos a flexión debe ser dispuesta de tal manera que las líneas de pega de los cantos queden horizontales y traslapadas (orientación de laminas tangencial) con el fin de evitar posibles delaminaciones y obtener una mayor capacidad de la sección. 
Teniendo en cuenta la comparación de la resistencia de diseño entre las maderas estructurales Andinas y los laminados de guadua, estos se proyectan como un material alternativo de ingeniería de fabricación industrial, con excelente resistencia y ambientalmente sostenible.

Los laminados de guadua para uso estructural no deben ser fabricados con adhesivo de polivinilo de acetato (PVA), debido a la inestabilidad de su resistencia al corte con el aumento en temperatura, debido a que este adhesivo es del tipo termoplástico.

Es recomendable realizar estudios de caracterización detallados con adhesivos tipo melaninaformaldehido u otros adhesivos termoestables con el fin de obtener laminados de guadua con resistencia a cambios de temperatura y humedad para poder garantizar una mayor durabilidad.

\section{REFERENCIAS}

American Standard Test Methods. 2007. ASTM-D143-94, Standard methods of testing small clear specimens of timber. Anual Book of ASTM Standards

American Standard Test Methods. 2007. ASTM-D198-07, Standard Test Methods of Static Tests of Lumber in Structural Sizes. Anual Book of ASTM Standards

American Standard Test Methods. 2007. ASTM-D905-07, Standard Test Method for Strength Properties of Adhesive Bonds in Shear by Compression Loading. Anual Book of ASTM Standards

American Standard Test Methods. 2007. ASTM-D 2395-07ae1, Standard Test Methods for Specific Gravity of Wood and Wood-Based Materials. Anual Book of ASTM Standards

American Standard Test Methods. 2007. ASTM-D3500-90, Standard Test Methods for Structural Panels in Tension. Anual Book of ASTM Standards

American Standard Test Methods. 2007. ASTM-D1037-94, Standard Test Methods for Evaluating Properties of Wood-Base Fiber and Particle Panel Materials// Anual Book of ASTM Standards

Asociación Colombiana de Ingeniería Sísmica. 1997. NSR-98: Normas Colombianas de Diseño y Construcción Sismo Resistente, Ley 400 de 1997, Decreto 33 de 1998

Barreto, W. 2003. Evaluación de la guadua laminada pegada, aplicada a propuesta de reticulado plano. Bogotá: Tesis Universidad Nacional de Colombia, Facultad de Arquitectura. Tesis para optar por el título de Arquitecto. $116 \mathrm{p}$

Durán, L. M. 2003. Estudio de guadua laminada y su aplicación al sistema tensegrity. - Bogotá: Tesis Universidad Nacional de Colombia, Facultad de Arquitectura, tesis para optar por el título de Arquitecto, $224 \mathrm{p}$

Gómez, C.; Rubio, F. 1990. Esfuerzos de trabajo para elementos estructurales de guadua. - Bogotá: Tesis Pontificia Universidad Javeriana, Facultad de Ingeniería, Departamento de Ingeniería Civil. Tesis para optar por el título de Ingeniero Civil: $157 \mathrm{p}$ 
International network for bamboo and rattan. 2007. Annual report. [www.inbar.int]. URL http:// www.inbar.int/publication/txt/ar2007.pdf. 02.05.2007

Junta del Acuerdo de Cartagena. 1984. Manual de diseño para maderas del grupo Andino. - Lima : JUNAC.

Lee, A.W.C.; Bai, X.; Bangi, A.P. 1998. Selected properties of laboratory-made laminated-bamboo lumber. Holzforschung 52 (2): 207-210.

Lopez, L. F.; Silva, M. F. 2000. Comportamiento sismorresistente de estructuras en bahareque. Manizales : Tesis Universidad Nacional de Colombia, Departamento de Ingeniería y Arquitectura, Tesis para optar por el título de Ingeniero Civil, 139 p

Lopez, L. F.; Trujillo, D. 2002. Diseño de uniones y elementos en estructuras de guadua. - Pereira : memorias taller avances en la investigación sobre guadua. Universidad Tecnológica de Pereira.

Martin, J. W.; Mateus, L.R. 1981. Resistencia a la compresión paralela a la fibra de la guadua de Castilla. - Bogotá : Tesis Universidad Nacional de Colombia. Facultad de Ingeniería, Tesis para optar por el título de Ingeniero Agrícola, $167 \mathrm{p}$

Ministerio de Agricultura y Desarrollo Rural de Colombia. 2006. Estadísticas cadena de la guadua en Colombia - Bogotá: Ministerio de Agricultura y Desarrollo Rural de Colombia. 28 p

Norma Técnica Colombiana. 2006. NTC-290, Maderas, Determinación del peso específico aparente, Instituto Colombiano de Normas Técnicas (ICONTEC).

Norma Técnica Colombiana. 2006. NTC-206-1, Maderas. Determinación del contenido de humedad para ensayos físicos y mecánicos, Instituto Colombiano de Normas Técnicas (ICONTEC).

Norma Técnica Colombiana. 2006. NTC-2279, Adhesivos. Determinación de la resistencia al cizallamiento por carga compresiva de uniones adhesivas, Instituto Colombiano de Normas Técnicas (ICONTEC).

Norma Técnica Colombiana. 2006. NTC-3377, Maderas. Ensayos con probetas pequeñas. Instituto Colombiano de Normas Técnicas (ICONTEC).

Norma Técnica Colombiana. 2006. NTC-663, Maderas. Determinación de la resistencia a la flexión, Instituto Colombiano de Normas Técnicas (ICONTEC).

Norma Técnica Colombiana. 2006. NTC-961, Maderas. Determinación de la tracción perpendicular al grano., Instituto Colombiano de Normas Técnicas (ICONTEC).

Norma Técnica Colombiana. 2006. NTC-784, Maderas. Determinación de la resistencia a la compresión axial o paralela al grano, Instituto Colombiano de Normas Técnicas (ICONTEC).

Norma Técnica Colombiana. 2006. NTC-785, Maderas. Determinación de la resistencia a la compresión perpendicular al grano, Instituto Colombiano de Normas Técnicas (ICONTEC). 
Norma Técnica Colombiana. 2006. NTC-775, Maderas. Determinación de la resistencia al cizallamiento paralelo al grano., Instituto Colombiano de Normas Técnicas (ICONTEC).

Nugroho, N.; Ando, N. 2001. Development of structural composite products made from bamboo II: fundamental properties of laminated bamboo lumber. The Japan Wood Research Society. Journal Wood Sci 47 (3): 237-242

Nugroho, N.; Ando, N. 2000. Development of structural composite products made from bamboo I: fundamental properties of bamboo zaphyr board. J. Wood Sci. 46 (1): 68-74.

Prieto, E.; Sánchez, J. 2002. Comportamiento de la guadua angustifolia sometida a flexión. - Bogotá: Tesis Universidad Nacional de Colombia. Facultad de ingeniería, Tesis para optar por el título de Ingeniero Civil, 139 p. 